rर्वि

1941-2016

$75_{\text {años }}$

RMA

Antropología Social

\section{Equívocos semánticos sobre transculturación y vacilaciones disciplinarias}

\author{
Semantic misunderstandings on transculturation and \\ disciplinary hesitation
}

Claudia Gilman

\begin{abstract}
Resumen
Este ensayo contribuye a la arqueología del concepto de transculturación, su empleo y su actualidad. Esa meta implica resumir los debates antropológicos entre los años treinta y cuarenta y analizar el uso del concepto "transculturación" - hasta ahora no señalado - en el pensamiento de Carlos Real de Azúa, mediado por el clasicista Gilbert Highet. Intento demostrar cómo se ha podido emplear transculturación y "aculturación" como sinónimos en determinados contextos humanísticos. Por último ensayaré las razones de la "desaparición" de la trayectoria de Fernando Ortiz, deudora de la lectura de Ángel Rama. La duda sobre el valor heurístico de la categoría transculturación, finalmente, es un medio eficaz para comprender algunos debates actuales entre antropología y estudios culturales.
\end{abstract}

Palabras claves: transculturación; antropología y estudios culturales; arqueología del saber; historia intelectual.

\begin{abstract}
This essay contributes to the archeology of the concept of transculturation, its use and its relevance nowadays. This goal implies summarizing the anthropological debates between the thirties and forties and analyzing the use of the concept "transculturation" - until now not mentioned - in the thought of Carlos Real de Azúa, mediated by the classicist Gilbert Highet. I aim to demonstrate how transculturation and "acculturation" have been used as synonyms in certain humanistic contexts. Finally, I will assay the reasons for the "disappearance" of the trajectory of Fernando Ortiz, debtor of the reading of Angel Rama. The doubt about the heuristic value of the category of transculturation, is an effective means to understand some current debates between anthropology and cultural studies.
\end{abstract}

Keywords: transculturation; anthropology and cultural studies; archaeology of knowledge; intellectual history.

Este ensayo contribuye a la arqueología del concepto de transculturación, su empleo y su actualidad. Esa meta implica resumir los debates antropológicos entre los años treinta y cuarenta y analizar el uso del concepto "transculturación" -hasta ahora no señalado-en el pensamiento de Carlos Real de Azúa, mediado por el clasicista Gilbert Highet. Intento demostrar cómo se ha podido emplear transculturación y "aculturación" como sinónimos en determinados contextos humanísticos, reconociendo sin lugar a dudas que "transculturación" es el que mejor describe los procesos surgidos de los contactos de culturas. Por último ensayaré las razones de la "desaparición" de la trayectoria de Fernando Ortiz, deudora de la lectura de Ángel Rama, para tratar de comprender algunos debates actuales entre antropología y estudios culturales.

\section{Contrapunteo antropológico de la aculturación y de la transculturación}

En los primeros decenios del siglo XX el pensamiento antropológico ya había demostrado la significación básica del trabajo de campo y el papel decisivo de la convivencia temporal con las sociedades humanas que se pretendía conocer. Se debatían los alcances y limitaciones teóricas del evolucionismo y el difusionismo como iniciales corrientes del quehacer antropológico, y se reconocía el sentido relativo de cada cultura respecto de otra, independientemente de su grado de desarrollo. Sin embargo, como examina Jesús Guanche (1995, pp.1-3), las categorías disponibles resultaban insuficientes para interpretar toda la riqueza y complejidad de los contactos interculturales y sus transformaciones. 
Entre los años 30 y comienzos de los 40, la antropología experimentaba guerras disciplinarias entre la escuela norteamericana y la británica. Una de sus divergencias se centraba en la importancia metodológica concedida a la historia de los pueblos nativos. La británica predominantemente funcionalista-era más empírica y defendía el trabajo de campo como el método más apropiado para las investigaciones, si bien la norteamericana también estimuló el trabajo de campo como instancia decisiva para la legitimación de y en la disciplina. La norteamericana daba más importancia al estudio de la historia, incluso la conjetural. La británica acusaba a la norteamericana de etnocentrista, y ésta a la británica de colonialista. Mientras los norteamericanos desarrollaban su concepto de aculturación, los británicos preferían hablar de contactos culturales. Las dos escuelas se enfrentaban también en torno a la cuestión de cuál de ellas trataban mejor al "nativo" (Santí 2002, p. 72). Tal vez pueda considerarse éste como un debate pionero de los que más tarde se establecerán en torno a la "subalternidad".

Ante la cantidad de estudios y definiciones contradictorias sobre la aculturación, el Social Science Research Council de los EE.UU designó una comisión ad hoc, presidida por Robert Redfield e integrada por Ralph Linton y Melville Herskovits. En 1936 y en breves cuatro páginas se publicó el "Memorandum for the Study of Acculturation". Puesto que "transculturación" (el libro de Ortiz no fue traducido al inglés hasta 1947) no tuvo en la antropología el reconocimiento casi desmesurado que encontraría en los estudios culturales y literarios de América Latina, conviene enmarcar lo que se discutía en la antropología. No se trataba solamente de definir la aculturación sino establecer también todo lo que no le concernía. Así, el "Memorandum" define afirmativamente la aculturación como aquellos fenómenos "que resultan cuando el contacto continuo y directo de grupos o individuos produce cambios en los patrones culturales de uno o ambos grupos" (Redfield et al. 1936, p.149); y por la negativa: "En esta definición, la aculturación debe distinguirse del cambio cultural del cual es sólo un aspecto y de la asimilación, que a veces es una fase de la aculturación. También debe diferenciarse de la difusión, que, si bien ocurre en todas las instancias de la aculturación, es no sólo un fenómeno que se da con frecuencia sin que se produzca el tipo de contactos especificados en la definición precedente sino que constituye solamente un aspecto del proceso de aculturación" (ibídem, p.150). El carácter indeterminado y la apertura del tema eran tan amplios que la comisión invitaba a los autores que lo trabajaban a que le enviaran sus obras para colaborar con la tarea de definición (ibidem, p.149).

Nada más lejos de la intención de Fernando Ortiz que enviar su trabajo en curso para ser examinado por aquella comisión ad hoc. Ortiz ya tenía pensado que no había mejora alguna para la aculturación y que debía reemplazarse por su propia categoría: la "transculturación". Es por eso que en Contrapunteo cubano del tabaco y el azúcar Ortiz provee su muy extensa definición que todavía hoy se cita ampliamente. Se desprende de ella que "transculturación" expresa mejor las diferentes fases del proceso transitivo de una cultura a otra, porque éste no consiste solamente en adquirir una distinta cultura, que es lo que en rigor indica la voz anglo-americana acculturation, sino que el proceso implica también necesariamente la pérdida o desarraigo de una cultura precedente, lo que pudiera decirse una parcial desculturación, y, además, significa la consiguiente creación de nuevos fenómenos culturales que pudieran denominarse de neoculturación.

Uno de los problemas de la aculturación estaba más allá de la definición y se presentaba en el terreno de la praxis. Una cosa era lo que estaba escrito y otra bien diferente lo que se hacía en el "estudio de campo". Hemos visto que las definiciones del memorándum y la de Herskovits incluían los fenómenos de contacto cultural entre dos o más culturas. Aunque la definición establece que el proceso involucra el contacto de culturas en un mismo nivel de importancia, la realidad era que los trabajos que se servían de esa herramienta conservaban, en los hechos la idea de la superioridad de la cultura observante respecto de la observada. Estas diferencias entre teorías y prácticas han sido recogidas y discutidas exhaustivamente por Martí Carvajal (2010).

\section{Prólogo de Malinowski a Ortiz: la burla en serio}

Cuando Malinowski y Ortiz se conocen en La Habana (fue en 1939 aunque las ediciones de Biblioteca Ayacucho y la de Ariel continúan el error de situarlo en 1929, afectando la progresión precisa de las influencias, problemas y la suerte del concepto en cuestión), el debate antropológico entre las mencionadas escuelas estaba en su cenit. En ese contexto Ortiz solicitó a Malinowski un texto que avalara su "transculturación". Son distintas las razones por las que ambos sabios coincidieron en la oposición al término aculturación. En el caso de Ortiz, como vimos, su objeción era la imprecisión conceptual del término. Enteramente posible, además, es que a esa objeción se uniera una crítica soterrada a los trabajos de Herskovits, principal promotor del término, a causa de sus trabajos de los años treinta sobre el negro en el nuevo mundo, donde ni siquiera se menciona el nombre de Ortiz. (Martí Carvajal 2010, p.15) En el caso de Malinowski, el resentimiento contra Herskovits y por extensión contra la escuela norteamericana de antropología, era harto evidente, como muestra Santí (2002, p.70).

Malinowski narra sus conversaciones, simpatías mutuas y entendimiento intelectual. La categoría aculturación nació y prosperó hasta donde se pudo, en el ámbito de la antropología estadounidense. Por esa razón, en 
su prólogo a Contrapunteo..., Malinowski acusa al concepto por su imprecisión, equivocidad y sus matices imperialistas, dando a entender abiertamente que la escuela norteamericana de antropología ha engendrado una categoría, "la palabra acculturation" que "amenaza con apoderarse del campo, especialmente en los escritos sociológicos y antropológicos de los autores norteamericanos" (Malinowski 1940, p. 4).

Ortiz desafiaba a Herskovits y su teoría de la aculturación en su alianza con Malinowski. Como árbitro de la polémica entre aculturación y transculturación, Malinowski aprovechaba la ocasión presentada por la edición de la Ortiz para atacar abiertamente a su rival, y de paso, inscribir un poco arbitrariamente, a Ortiz dentro de su propia corriente teórica. El prólogo de Malinowski tiene mucho de pieza humorística. En el breve escrito, Malinowski declara que la voz acculturation contiene un conjunto de determinadas e inconvenientes implicaciones etimológicas y que es un vocablo etnocéntrico con una significación moral. No le basta a Malinowski criticar la implicación etnocentrista de la"acculturation". También se opone al vocablo por su ingrata fonética, afirmando que "suena como si se arrancara de un hipo combinado con un regüeldo" (ibídem, p. 4). Otra broma, sin duda, es citarse a sí mismo para demostrar cuánto lo convence la terminología de Ortiz, que por supuesto no existía cuando Malinowski escribía el trabajo, un gesto que recuerda al "Kafka y sus precursores" argumentado por Jorge Luis Borges. ¿Será también una broma bautizar a Ortiz como funcionalista?

Otro problema del prólogo de Malinowksi es que comenta que antes de conocer a Ortiz ya lo habían impresionado sus "estudios de los aspectos económicos, sociales y culturales que ofrecen los recíprocos influjos los africanos y los latinoamericanos". Esta homologación de "africanos" y "latinoamericanos" está fuera de los razonamientos de Ortiz. Malinowski no supera el "colonialismo mental." Los "latinoamericanos" no son una categoría como aparecen en Malinowski y tampoco son tema de Ortiz. Queda a cargo de Malinowski referirse dos veces a esa dudosa contigüidad en su prólogo. En cuanto a lo terminológico (la "transculturación") los colegas están contestes sobre la mayor felicidad léxica expresada en el término transculturación, hecho que reconoce el propio Herskovits en la carta que le manda a Ortiz y en la que también acusa la recepción del libro, que Ortiz le envió (transcripta en Santí 2002, pp. 262263). Sin duda, la sensibilidad lingüística no es un asunto menor ya que llegó a preocupar a José María Arguedas él también antropólogo en su tan citado discurso al recibir el premio Inca Garcilaso aseveró "no soy un aculturado", enunciado cuyos avatares serían discutidos como plantilla conceptual por Ángel Rama y sus seguidores literarios. ${ }^{1}$

\footnotetext{
1 Véase José María Arguedas, "No soy un aculturado", Marcha 1427 (1968), Amaru N6 (abril/junio1968), "Primer diario El zorro de arriba y el zorro de abajo", El comercio (1 de junio de 1969),
}

En los trabajos sobre la transculturación suele citarse siempre a Rama y a su fuente, Ortiz. Casi no ha sido señalado el recorrido que lleva al crítico cultural uruguayo, Carlos Real de Azúa que permite revisitar la arqueología del término.

\section{Real de Azúa: senderos que no se bifurcan}

En dos ocasiones (la primera en un texto de 1959, la segunda en un texto póstumo; ambos registrados en las Obras citadas), al menos, se registra en Real de Azúa el uso de "transculturación" y en ambos casos el concepto hace referencia a procesos de contactos culturales, de los cuales da por sentado surgirán transformaciones tanto de las ideas como de las prácticas que las conciernen. Es más, no existe ningún contacto cultural que no derive en transformaciones y "transculturaciones", nombre que da Real de Azúa a los resultados de esos contactos. A esa ceguera para comprender el carácter abierto e impredecible de la historia la denomina "enfermedad infantil del antimperialismo" y así lo concebirá en los dos casos. Real de Azúa utiliza, de manera heurística y desafiante la "transculturación".

En un contexto de "paridad" intelectual y "disparidad" "de fortuna, origen e importancia estratégica mundial, la transculturación es un factor por un lado inevitable, en lo que compete a la mecánica misma del proceso; y por otro, una herramienta que puede usarse a favor de los menos favorecidos. Real de Azúa se ubica en el campo de la historia de las ideas y, dada su perspectiva, descarta la posibilidad de imposiciones mecánicas. En parte porque los tiempos han cambiado y reducido la brecha alguna vez inconmensurable en el estado de desarrollo de las diversas sociedades y/o culturas. Real de Azúa definirá la transculturación como corolario lógico respecto de la ambigüedad de los procesos intelectuales y a su poder. Postula la capacidad de resistencia contra la idea de que las culturas son inertes ante la penetración ideológica. En contra de quienes sólo denuncian pero no intentan la acción ni comprenden demasiado lo que denuncian, Real de Azúa se sitúa contra quienes piensan que la política local es sólo un complot externo o una traición interna contra la que nada se puede hacer.

Con la volanta "La rueda en el aire" y el título "Liberalismo y principismo", en 1959 Real de Azúa comenta un libro, cuyo título omite mencionar, en el que analiza la encarnación uruguaya del liberalismo en el grupo de los "letrados principistas". ${ }^{2}$ En la reseña, Real de Azúa

\footnotetext{
"Inevitable comentario a unas ideas de Julio Cortázar", Marcha (30 de mayo de 1969), y Julio Cortázar "Un escritor y su soledad", Life en español (7 de abril de 1969). Estos textos han sido reproducidos en varias compilaciones de ambos autores. Otros transculturadores que anteceden, son coetáneos o surgen después del concepto son Mariano Picón Salas, Félix Schwartzman y Lauro Ayestarán.

2 El libro seguramente es La Universidad de la República en la formación de nuestra conciencia liberal, de María Blanca Paris de Oddone. Los principistas fueron un grupo de ilustres jóvenes
} 
confirma que la denunciada "penetración cultural" no tiene el mordiente necesario para oponerse a fuerzas intelectuales contrarias. Por eso critica los lamentos y la "caudalosa versión en la ensayística histórica hispanoamericana" que sólo ve en el liberalismo la máscara histórica del imperialismo. En primer lugar sitúa al liberalismo en el contexto de su aparición, recordando que enfunda valores que no han perimido, aunque "hoy los veamos o los deseamos en otros contextos y ante otras circunstancias". Y si cuando encarnó entre los principistas, argumenta Real, "ese liberalismo no pasó de interminables loas a la Libertad y al irrestricto arbitrio individual" (Real de Azúa 1959, p.22), lo hizo en una sociedad sin estructuras, sin formalidades.

Real de Azúa argumenta que la costumbre uruguaya de privilegiar lo ideológico por sobre lo material, y la perspectiva de lo genérico y lo abstracto en vez de lo concreto,

"son también atributos de nuestra mentalidad liberal y no sólo de esa variedad más de penetración imperialista. Y esto lo es por cuanto los fenómenos de aculturación y transculturación (el contacto y la interpenetración de culturas) como lo observaba en un brillante ensayo Gilbert Highet, son uno de los hechos más constantes de la historia universal y cubren un área mucho más ancha de lo que hoy se entiende, bajo el estímulo marxista, por imperialismo" (Real de Azúa 1959, p.20).

Como dirá luego, es la humanidad la que hace la historia y no sólo es la lucha de David y Goliat. Según Real de Azúa, "los hombres son casi siempre, más allá de su consciente querer, instrumento de fuerzas, de significaciones que los desbordan" (ibídem, p.70), aclarará bien en la segunda ocasión en que trate sobre la transculturación en un libro escrito entre 1961 y 1963 pero que se editó póstumamente. Allí opone la transculturación al presente inevitable de la penetración ideológica. Esta vez critica los temores de sus pares, sus compañeros de ruta y realiza una intervención sobre el presente que, paradójicamente dejó inédita. En lugar de sólo denunciar la permanente máquina de soborno y seducción sobre todos los sectores decisivos de los países atrasados por medio de invitaciones y becas, Real de Azúa se pregunta "¿Hasta qué punto los contactos humanos que promueven no llevan imprevisiblemente -en ambas direcciones-gérmenes y sugestiones adversas para los que tales tratos promueven (Real de Azúa 1996, pp. 74-75). Es un error entender todos los contactos de cultura, todos los fenómenos de

universitarios uruguayos que rechazaron las divisas partidarias, unidos por ciertos principios que creían incontrovertibles. Su ideología, si bien bastante heterogénea, tenía algunos puntos en común: abogaban por la aplicación del liberalismo económico, apoyaban la creación de partidos basados en las ideas, a blancos y colorados y, sobre todo, a sus respectivos caudillos. aculturación como "imperialismo".

Como se ve, la transculturación llega a Real de Azúa del "brillante ensayo de Gilbert Highet" cuyo título tampoco proporciona. Habrá que leer y descartar antes de encontrar. Para Highet, reconocido autor de la monumental The Classical Tradition: Greek and Roman Influences on Western Literature (1949) y traductor al inglés de la imprescindible Paideia, de Werner Jaeger, ese "brillante ensayo" es uno sus muchos libros "menores". Habrá que repasar título por título las publicaciones del ensayista y profesor escocés, hasta encontrar la fuente de Real de Azúa: The Migration of Ideas, surgido de las conferencias dictadas en la North Foundation at Franklin and Marsall College, 18 y 19 de marzo de 1954, conocido en castellano con un título menos interesante y menos pertinente: La fuerza de las ideas (la edición en inglés fue publicada en 1954 por Oxford University Press, en castellano por la Editorial Hispano-Europea de Barcelona, en 1956).

\section{Gilbert Highet, intruso e inesperado}

En Highet encontró Real de Azúa la herramienta de su transculturación. Pero ¿dónde la encontró Highet? Citada como "Conclusión" del libro The Migration of Ideas, la bibliografía que ha servido a Highet pertenece por completo a la escuela estadounidense de antropología, como lo indican los títulos y los nombres de los autores: De allí se puede inferir que ha llegado a la noción de "transculturación" partiendo de la cuestionada "acculturation".

En su propuesta a favor de una historiografía consistente, Highet demuestra que es imprescindible el estudio de las ideas y de los procesos que condujeron a ellas; y recurre a los conceptos de migración de las ideas o adquisición de valores culturales a través de la educación. El desarrollo de su argumentación es sumamente sugestivo, válido para el estudio de todo tipo de culturas y también para los diversos tiempos de la historia, incluyendo los contactos culturales del presente. Si bien es cierto que desde su perspectiva comparatista Highet está acostumbrado a analizar la presencia de culturas pasadas en las que le son posteriores, en particular la relación de la tradición grecoromana, se preocupa por averiguar cómo circulan las ideas en la historia, en el pasado y en el presente y -nada lo impide, al menos en el terreno de las hipótesis-hacia el futuro. Evitando abordar la historia sólo desde el punto de vista político y económico, que resulta insuficiente para elaborar el relato histórico, Highet plantea que es preciso preguntar y responder cuál es el papel de las ideas sobre los actos humanos y cómo se desarrolla y tiene lugar la difusión de aprendizajes entre grupos diferentes de personas.

Highet entiende además que los estudios humanos se encuentran siempre en movimiento, expandiéndose 
y comprimiéndose, invadiendo territorios ajenos, expresando nuevos propósitos, definiendo temas. La historia que registra los hechos del pasado ignora cuáles de ellos serán documentos para escribir la del futuro. En el tiempo que lleva preguntarse qué hechos pasados estudia la historia se habrán producido millones de acontecimientos. Algunos parecerán triviales en un cierto momento, en diez o cincuenta años, otros cobrarán tal importancia para la existencia de millares de seres humanos, que habrán adquirido categoría histórica. Sin embargo, la aniquilación de poblaciones nativas en América carece para él de aristas problemáticas: los ritos católicos son mejores que los ritos de los salvajes aztecas. Fuera de la valoración subjetiva y partiendo de que Highet es más optimista para considerar las transculturaciones del pasado que las que están en curso en el presente y con pronóstico de empeorar hacia el futuro, lo que importa subrayar es que para Highet esos procesos son continuos e inevitables.

Un ejemplo proporcionado por Highet-además en la línea del Contrapunteo... de Ortiz - es la historia mundial de los cultivos, inventos absolutamente humanos. Existe sólo una docena de lugares en los que las plantas cultivadas crecieron originariamente en estado silvestre. Pero desde allí, se han extendido por el resto del mundo, cambiando la existencia de las gentes. Finalmente, gracias a la humilde papa se escribe todo un capítulo de la historia general y colectiva. Highet postula que cualquier estudio detallado de la conexión entre culturas y migración de ideas, deberá, sin duda, tomar en consideración cierto ángulo de convergencia respecto a lo mucho o poco que tengan en común las dos culturas a considerar. La noción de "ángulo de convergencia" para operar con la categoría de "transculturación" es una invención que Highet no reclama como mérito.

\section{Traductores transculturadores}

En 1948 se publica la primera edición en inglés de El hombre y sus obras. La ciencia de la antropología cultural, de Herskovits, que traducida al español desde 1952 hacia 1995 ya contaba con once reimpresiones por el Fondo de Cultura Económica. En estas versiones, el capítulo XXXI está dedicado a la Transculturación: la transmisión cultural en marcha; pero ello no significa la aceptación terminológica, sino que su traductor $\mathrm{M}$. Hernández Barroso, conocedor del neologismo, aclara que "La hemos venido usando en este libro, como la traducción más acertada de acculturation, así como en otras obras anteriormente editadas por Fondo de Cultura Económica". Asimilando los términos aparentemente antagónicos en una sola palabra, es una decisión editorial que ocluye en sus propios términos una querella científica.

Por otra parte, el libro de Highet contó con un traductor atento a lo que estaba leyendo. El carácter positivo de los procesos de acculturation habrá convencido a un buen lector, capaz de comprender que el fenómeno no podía representarse con un término "privativo". De modo que J.F. Yáñez, este traductor intuitivo, inventa su propio neologismo y escribe "culturación" cada vez que tiene que evitar el equivalente castellano de acculturation. En The Migration of Ideas, acculturation es sencillamente una categoría históricamente necesaria que, además, conlleva la idea de progreso. Highet parece seguro de que la culturación aumenta del poder de quienes están en medio de esos procesos, por otra parte inevitables e infinitos. Por eso le da un significado positivo.

La posición transculturadora de Real de Azúa, mientras tanto, lo lleva a formular hipótesis propias que se enfrentan a las de la mayoría de sus colegas en lo que concierne a la relación con los EE.UU. La apuesta por la transculturación es radical en Real de Azúa, ya que la utiliza en relación con el presente y la presenta como herramienta para neutralizar/desviar las estrategias de cooptación instrumentadas por los EE.UU.

Pese a que no es el caso, parecería que los usos y la significación de la transculturación propuestos por Highet están más cerca de Ortiz ya que más que ningún otro transculturador explica el proceso de "toma y daca" del que hablara Malinowski en el prólogo a Contrapunteo... Lo mismo sucede cuando da cuenta debidamente de las neoculturaciones que se dan tanto en la cultura observadora como en la cultura observada.

\section{Ortiz versus la transculturación}

Pese a los antagonismos del pasado entre Herskovits, Malinowski y Ortiz, las diversas variantes conceptuales podían verse en la práctica como alternativas complementarias. Al menos en la trayectoria que termina en Real de Azúa y que permite pasar de Herskovits a Highet. ¿Podríamos decir que si Rama fue por el camino de Swann hacia Ortiz y Malinowski, Real de Azúa fue por el camino de Guermantes a través de Highet y Herskovits y que como en la novela de Proust ambos caminos llevan al mismo lugar, en este caso, a la transculturación? Dice, al respecto Enrico Mario Santí:

"Acaso mi tesis más polémica es que la llamada confrontación entre "aculturación" y transculturación propuesta por Malinowski, acatada por Ortiz y esgrimida por sus admiradores, no se justifica del todo. Aun cuando sí se justifica la invención del término "transculturación" sobre todo en lo que toca a su justicia léxica-el concepto mismo resulta gratuito, o al menos equívoco, en relación a la "aculturación", su ostensible polo dialéctico. Deslindar el asunto me ha parecido necesario, no sólo en honor a la verdad, sino porque el equívoco sigue dando lugar a más de un argumento ideologizante dentro de los llamados estudios pos-coloniales 
que hoy hacen su agosto en la academia euronorteamericana" (Santí 2002, p.11).

Para Santí no está en duda la importancia de la tarea científica de Ortiz; todo lo contrario. Lo que cuestiona es cuán poco se reconocen los méritos de Ortiz y la mengua de su verdadero legado: no se trata sólo de reconocer la "invención" de un prefijo. Santí lamenta la innecesaria oposición entre transculturación y aculturación, de lo cual deriva su pregunta sobre las relaciones entre la antropología y los estudios culturales:

"Publico este libro en medio de discusiones intensas sobre los llamados "estudios culturales" su relación con la antropología y sociología, la teoría crítica, y los estudios literarios, sobre todo los que tratan la realidad pos-colonial y la hibridez cultural. En casi todos ellos la obra de Ortiz adquiere, no siempre sin razón, el parangón de precursor, sobre todo en lo que toca a la invención del manido término y concepto de "transculturación". No poco ha influido en ello, dentro de los estudios latinoamericanos, el libro de ensayos de Ángel Rama Transculturación narrativa en América Latina (1982) que adaptó el concepto a sus intereses literarios y lo volvió a poner en circulación. Confieso que mi relación con ese nuevo contexto es más bien polémica: discute y extiende sus conclusiones, pero critica los usos que a veces ha hecho de las ideas de Ortiz. En ese sentido, aporto aquí no tanto una lectura de las dudosas aplicaciones que Rama y sus discípulos hacen del término cuanto una arqueología de las ideas del propio Ortiz, que a veces contradice esas y otras aplicaciones interesadas." (Santí 2002, pp. 10-11).

Para Guanche, la propuesta de la transculturación tal como había sido formulada por Ortiz en 1940, no significó un simple cambio de prefijo sino que se derivó de una profunda reflexión basada en múltiples investigaciones durante más de tres décadas sobre la problemática etnosocial cubana y sus nexos internacionales. Sin embargo, el propio Guanche afirma que Ortiz no fue objeto de tanta divulgación ni aceptación internacional, lo que atribuye a múltiples factores, que tienen su raíz histórica en el propio desarrollo de la antropología norteamericana, en su alta capacidad divulgativa y su exigua disposición receptiva de otras antropologías nacionales. Por otra parte, sugiere que los antropólogos ingleses han operado con diferentes posiciones teóricas y han preferido el término "contacto cultural" al de aculturación, sin adoptar "transculturación" (Guanche 1995, p.6).

Según Martí Carvajal dos razones pueden explicar el poco predicamento del polígrafo cubano: una de ellas sería que al invitar a Malinowski a escribir la introducción de Contrapunteo..., Ortiz se colocó en el medio de una batalla académica y que por esa causa se lo haya asociado con la escuela británica, aunque admite que es difícil "afiliar el trabajo de Ortiz al funcionalismo" (Martí Carvajal 2010, p.15). La segunda pone el acento en presuntas diferencias políticas. Hay demasiados trabajos sobre Ortiz en los que se formulan hipótesis - constatándolasobre la falta de reconocimiento académico que se le debe. Guanche pone fecha de caducidad a la "transculturación" en los años 60, cuando el concepto de aculturación ha sido poco a poco sustituido en la literatura antropológica norteamericana por otros como "cambio cultural", "desarrollo" y "movimiento social". Lo que parece evidente es, como especula Santí, es que la "transculturación" se haya vuelto un concepto de Rama que opaca al propio Ortiz.

De manera que parecería que en las discusiones antropológicas sobre la aculturación y la transculturación Ortiz no ha tenido razón. Las categorías declinaron y fueron reemplazadas por otras, de modo que no hubo mayor interés en continuar estableciendo sus límites y significados. Hay quienes piensan, como Martí Carvajal que la categoría aculturación nació problemática y murió problemática. Dada su incierta semántica, en la década del cincuenta se volvió a reunir un comité para redefinir de una vez por todas, su valor heurístico. Para la década del 50, dice, se hizo evidente que la recolección de material empírico sobre aculturación avanzaba más rápidamente que los intentos teóricos para ordenar y codificar los conceptos centrales que brindarían a esos estudios sus máximos resultados (traducción mía) Un grupo de investigadores propuso realizar un seminario interuniversitario dedicado a la categoría de aculturación. De ahí surgió el ensayo "Acculturation: An Explanatory Formulation", de 1953 escrito por Siegel, Vogt, Watsn y Broom. Su definición de aculturación fue más corta y simple que la de 1936: Puede definirse como el cambio cultural iniciado por la conjunción de dos o más sistemas culturales autónomos, según Martí Carvajal (2010, p. 13).

En 1995 se realizó la Twelfth Annual Visiting Scholar Conference en la universidad de Southern Illinois para tratar el conflicto cultural a nivel mundial. Las ponencias de esta conferencia se publicaron bajo el título Studies In Culture Contact: Interaction, Culture Change, and Archaeology (1998). Es notable que en los veintiún ensayos que conforman la obra sólo el de la arqueóloga Kathleen Deagan mencionara a Ortiz y a Contrapunteo... De todos modos, a más de 70 años de la escritura del memorándum la categoría aculturación aun produce problemas. Ni el término ni el concepto han estado claros ante la comunidad académica (Martí Carvajal 2010, p. 13).

Pero si bien es cierto que por un lado la disciplina antropológica no reconoció a Ortiz como debía, su 
nombre saltó de disciplina y llegó a los "estudios culturales" donde apenas se le reconoce un ínfimo aporte. Ese "olvido", al que se refieren Santi y Martí Carvajal, sería un contrapunto entre la ausencia de reconocimiento por parte de la antropología y su inconmensurable fama en otro campo disciplinar, la literatura, extendido por Rama a la crítica literaria latinoamericana. Mientras Martí Carvajal, Guanche, Santí y muchos otros lamentan la pérdida de predicamento que sufrió Ortiz en el campo de la antropología, a otros sorprende cómo contrasta con la inmensa fama reconocida a Ortiz, en el campo de los estudios culturales. Los reconocimientos son de variada procedencia pero el que más atracción tuvo fue el que le tributó Ángel Rama a partir de sus hipótesis sobre la transculturación como "dispositivo" que resuelve en la literatura tensiones entre la tradición y los "impulsos modernizadores" y contribuye a producir una literatura de gran calidad, como la de Rulfo, Arguedas y Guimarães Rosa, según Rama.

Como el propio Rama no deja de aclararlo en su libro de 1982, este concepto procede del libro de Ortiz. Pero la prosa poética y las sugestivas ideas del cubano no son su única fuente. Antes de Rama, había conocido e incluso aplicado el concepto de Ortiz el musicólogo Lauro Ayestarán en un capítulo sobre la música de los negros en Montevideo dentro de su extensa e inconclusa obra La música en el Uruguay (1953). Vale consignar que Rama conoció y admiró la obra de Ayestarán, y pronunció palabras de encendido elogio en ocasión de la prematura muerte del investigador justo en la dirección del rescate de una cultura popular, buscada como nervio y tejido de la nueva cultura latinoamericana (cf. Rocca 2013). Si de Ayestarán pudo tomar esta referencia, es claro que a través de Darcy Ribeiro, otro crítico cultural que admiraba, Rama entró a los problemas de la antropología.

La de Ortiz es actualmente una fama excentrada quizás injustamente puesto que si los alcances de su influencia son enormes, las razones profundas de su lección quedan reducidas a su hipótesis sobre la transculturación. La constatación procede de Santí. Este especialista lamenta que el debido reconocimiento hacia Ortiz se restrinja al "manido término y concepto de transculturación". No menos se queja de la celebración de Ortiz por parte de los 'estudios culturales', su relación con la antropología y la sociología, la teoría crítica y los estudios literarios, sobre todo los que tratan la realidad pos-colonial y la hibridez cultural, en gran parte desatendiendo las contribuciones "nativas". Análogamente, un debate parecido entre las literaturas comparadas y los estudios culturales se ha planteado casi los mismos problemas desde mediados de los años noventa respecto a si las literaturas comparadas morirían en manos de los estudios culturales (cf. Gilman 1997).

Y tal vez sea otra vez afirmativa ante la pregunta de los antropólogos. En algunos de los casos, hay antropólogos que defienden a ultranza una alianza productiva con los estudios culturales, a los que atribuyen una cualidad de intervención política inexistente en otras áreas de pensamiento disciplinario.

La categoría de "transculturación" requiere un retorno razonado a la obra de Ortiz, una cabal comprensión de las operaciones reales de ida y vuelta que permiten proponer una nueva notación para un --¿nuevo?-objeto o concepto operativo. También habría que reponer históricamente las fases que llevan a la transculturación. Haría falta analizar comparativamente para establecer si la categoría es aplicable a otras zonas donde se han producido importantes y dramáticos contactos culturales o si acaso, la "transculturación" debe restringirse a estudiar las muchas peculiaridades de Cuba, llave de los mares, una isla siempre ardiente e hito histórico singular. La "transculturación" también debería funcionar como el verdadero ida y vuelta, toma y daca, implícitos de manera necesaria y explícita en la especulación de Ortiz.

Buenos Aires, agosto de 2016

\section{Bibliografia}

Gilman, C. (1997). "La literatura comparada: Informe para una academia (norteamericana)". Filología, Año XXX (1-2): 33-43.

Guanche Pérez, J. (1995). "Avatares de la transculturación orticiana". Temas (nueva época) n 4 : 121-128. Disponible en web: http://www.afrocuba.org/ Antol2/Book/Avatares\%20-\%20JG.pdf.

Malinowski, B. (1940). "Introducción". En Fernando Ortiz, Contrapunteo Cubano del Tabaco y el Azucar (Advertencia de sus contrastes agrarios, económicos, históricos y sociales, su etnografía y su transculturación). Jesús Montero, La Habana, pp. 3-10.

Martí Carvajal, A. (2010). "Contrapunteo etnológico: el debate aculturación o transculturación desde Fernando Ortiz hasta nuestros días". Kálathos 4 (2): 1-22.

Real de Azúa, C. (1959). "Liberalismo y principismo". Marcha 949: 20 - 22.

Real de Azúa, C. (1996). Tercera posición, nacionalismo revolucionario y Tercer Mundo. Una teoría de sus supuestos. R. Cotelo, Montevideo, vol. 1. Cámara de representantes República Oriental del Uruguay.

Santí, E. M. (2002). Fernando Ortiz: contrapunteo y transculturación. Editorial Colibrí, Madrid.

Redfield, R., Linton, R. \& Herskovits, M. J. (1936). 
"Memorandum for the Study of Acculturation". American Anthropologist 38: 149-152.
Rocca, P. (2013). "El bosque y el árbol. La contribución de Lauro Ayestarán a los estudios literarios". Casa de las Américas 273: 29-41. 